Original Research

\title{
Evaluation of aminoglycosides utilization in intensive care units of a teaching hospital in southern Iran
}

\author{
Afsaneh VAZIN ID, Mahtabalsadat MIRJALILI ID, Sara ASADI ID).

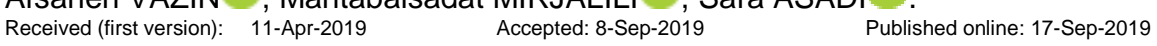

\begin{abstract}
Background: Inappropriate use of antimicrobial agents is one of the most important factors in inducing resistance and prolonged hospitalization as well as increase in patient mortality rate.

Objective: The aim of this study was to evaluate aminoglycosides (AGs) usage pattern at intensive care units (ICUs) of Nemazee hospital Shiraz, Iran.

Methods: In this cross-sectional study, the usage pattern of AGs was evaluated during 32 months. Guidelines for AGs usage were approved by the drug and therapeutic committee of the hospital, and criteria were developed to assess 11 parameters involving AGs therapy, such as proper indication for the use of the drug, dosage and duration of therapy. Clinical parameters, such as microbial culture and sensitivity, serum creatinine ( $\mathrm{SCr}$ ) and creatinine clearance, and white blood cell count were evaluated.

Results: Ninety-five patients were recruited, 50 male and 45 females. In most patients (64\%) the origin of infection was hospital and only in $36 \%$ of them, community was the source. Ventilator associated pneumonia $(27 \%)$, central nervous system (25\%) and urinary tract infection (10\%) were the most important indications for AGs prescription. Scores of AGs usage at Nemazee hospital was calculated as 5.9 out of 11 , which meant that in only $54 \%$ of cases AGs prescription was based on guideline proposed by the Department of Clinical Pharmacy of Nemazee Hospital.

Conclusions: Non-adherence to the guidelines occurred frequently in the ICUs of Nemazee hospital. Prescription of loading dose, and AGs level measurement were not done and evaluating microbiological data was often neglected. Incorporating pharmacists in the health care team and holding training programs for physicians and nurses with the goal of raising awareness about the proposed guideline.
\end{abstract}

\section{Keywords}

Aminoglycosides; Inappropriate Prescribing; Drug Utilization; Professional Practice; Guideline Adherence; Creatinine; Pharmacy Service, Hospital; Intensive Care Units; Pharmacists; Cross-Sectional Studies; Iran

\section{INTRODUCTION}

Inappropriate antibiotic prescription can increase the duration of hospitalization and mortality. ${ }^{1-4}$ Inappropriate use and dosing of antibiotics can lead to antimicrobial resistance. Increasing antimicrobial resistance by itself is a reason for increased usage of AGs. On the other hand, improper dosing of AGs can lead to resistance, adverse drug reactions, such as nephrotoxicity and ultimately treatment failure. ${ }^{5}$ Hospital-acquired infection (HAI) is defined as infections that develop after 48 hours of hospitalization, which did not exist at the time of admission. HAl is associated with increasing medical costs, duration of hospitalization, complications, and increasing morbidity and mortality. ${ }^{6}$

Antibiotic misuse has led to increased adverse effects, drug resistance and the outburst of multidrug resistant (MDR) organisms. ${ }^{7,8}$ To reduce medication cost and to control the prevalence of antibiotic-resistant bacteria in the community, there is no doubt that physicians must optimize the use of antibiotics. Pharmacists can play a pivotal role in order to reach this objective. ${ }^{11}$ Drug Utilization Evaluation (DUE) is a method to understand the

Afsaneh VAZIN. Department of Clinical Pharmacy, Faculty of Pharmacy, Shiraz University of Medical Sciences. Shiraz (Iran). vazeena@sums.ac.ir

Mahtabalsadat MIRJALILI. Department of Clinical Pharmacy, Faculty of Pharmacy, Shiraz University of Medical Sciences. Shiraz (Iran).mahtab.mirjalili@gmail.com

Sara ASADI. Department of Clinical Pharmacy, Faculty of

Pharmacy, Shiraz University of Medical Sciences. Shiraz (Iran). sarah.asadi70@yahoo.com drug administration problems and to see if drugs are administrated appropriately. ${ }^{12}$ It is also a tool to optimize the use of antibiotics. ${ }^{13}$ Several studies were conducted in this regard. ${ }^{11,14,15}$

Early and appropriate treatment of patients in intensive care unit (ICU) is critical when managing infections, which could help to reduce mortality rates in patients with severe sepsis or septic shock. ${ }^{16,17}$ Aminoglycosides (AGs) are one of the most essential antibiotics used in ICUs. ${ }^{18}$ AGs are suggested as an adjunct to extended spectrum betalactams by surviving sepsis campaign (SCC) international guidelines to manage sepsis and septic shock. ${ }^{19}$ Therefore, AGs are often given as part of empirical therapy for severe sepsis and septic shock, especially when Gram-negative bacteria are suspected. ${ }^{20}$

Several studies showed suboptimal AG in the early phase of therapy in critically ill patients. ${ }^{21-23}$ In Iran, it has become a routine practice to prescribe $A G$ in combination with betalactam antibiotics in severe gram-negative infections.

As improper usage and dosage of antibiotics can lead to antimicrobial resistance, DUE as a tool to detect the antibiotics utilization flaws can lead to optimization of the antibiotic administration by reducing resistance. As far as we know, no study was conducted on aminoglycoside utilization in Iran; hence, the present study focused on AGs usage pattern based on global standard drug consumption in ICUs to optimize its administration and to reduce drug resistance to this antibiotic in a referral hospital in southern Iran. 


\section{METHODS}

The usage pattern of AGs (Amikacin, Gentamicin) was evaluated in a prospective study during 32 months from January 2015 to August 2017 on patients admitted to 4 (internal, general, central, and surgical) ICUs at Nemazee hospital, a general multispecialty, referral, tertiary healthcare setting affiliated with Shiraz University of Medical Sciences, Iran. The Institutional Review Board and the Medical Ethics Committee of the hospital approved the study protocols. Written informed consent was obtained from all participating patients or their guardians.

All hospitalized patients in ICUs who had received at least three consecutive fixed-dose of AGs (to reach to the steady state concentration) were included in this study without considering age and gender. Those who had received less than three consecutive fixed-dose of $A G$ due to ward transfer, hospital discharge, or death were excluded. Data gathering was done by a general pharmacist under the supervision of a senior clinical pharmacy attending.

General demographic information, such as age, gender, height, total body weight, body mass index, date of admission and discharge were filled in the case report format. The patient's medical history, diagnosis, reason for patient's referral to the ward, final diagnosis, pre-existing medical conditions, and whether the patient's infection was acquired from the community or hospital, were recorded. Laboratory data consisting of microbiological culture, hematologic parameters, including white blood cell (WBC), biochemical data, including blood urea nitrogen, serum creatinine, and immunologic factors (procalcitonin, c-reactive protein) were also recorded before and during the course of $A G$ treatment.

To evaluate AG usage, a local guideline proposed by the Department of Clinical Pharmacy of Nemazee Hospital based on European guideline was used. ${ }^{24}$ The guidelines were intended for use after adaptation to local resistance data by local therapeutic and drug committee. As an objective, eleven indexes were taken into account. Each index was scored as either 0 or 1 based on whether the index was evaluated as inappropriate or appropriate, respectively. A sheet, which consisted of eleven indexes, was completed for each patient. By adding up the scores for each index, the total score was determined for each patient, and at the end the mean was calculated.

These items are as follows: 1) Administration of loading dose, 2) Route of administration, 3) Dosing method, 4) Appropriate dose, 5) Indication, 6) Dose readjustment if necessary (including reduction of renal function), 6) Evaluation of patient's serum creatinine and blood urea nitrogen levels before prescription, 7) Assessment of patients' serum creatinine levels periodically, 8) Evaluation of microbial culture from the suspicious site of infection before prescription dosing method, 9) Evaluation of microbial cultures 48-72 hours after prescription, 10) Discontinuation or reduction in dose of $A G$ in patients with AG nephrotoxicity (a rise in serum creatinine by more than $0.3 \mathrm{mg} / \mathrm{dL}$ within 48 hours, a rise in serum creatinine increased to more than 1.5 times baseline within the last 7 days or urine output less than $0.5 \mathrm{~mL} / \mathrm{kg} / \mathrm{hr}$ for more than 6 hours), 11) An increase in dose or a change in the type of antibiotics in the case of inappropriate responses to treatment. ${ }^{25}$ Appropriate dosing method was considered as $\mathrm{mg} / \mathrm{kg}$ dosing of the AG based on calculated $\mathrm{CrCl}$. It is also worth mentioning that the patients' response to treatment was assessed based on fever, white blood cells count, microbial cultures, level of consciousness, radiological images, and clinical features. Computerized physician order entry (CPOE) was used in the ICUs as an electronic prescribing system.

Statistical analysis was performed by SPSS) version 20 . Continuous and discrete variables were reported as mean, standard deviation and percentage, respectively. Comparison between numerical variables between two groups was performed, using independent t-test.

\section{RESULTS}

In this study 95 patients with the mean age of $40 \pm 18.2$ were screened for 963 days. Forty-seven percent of the study population were women. Patients' demographic data are shown in Table 1.

\begin{tabular}{|c|c|}
\hline \multicolumn{2}{|l|}{ Age (years) } \\
\hline $\begin{array}{r}\text { Mean (SD) } \\
\text { Range }\end{array}$ & $\begin{array}{c}56.7(18.2) \\
2-92\end{array}$ \\
\hline \multicolumn{2}{|l|}{ Gender, n (\%) } \\
\hline $\begin{array}{r}\text { Male } \\
\text { Female }\end{array}$ & $\begin{array}{l}45(47 \%) \\
50(53 \%)\end{array}$ \\
\hline \multicolumn{2}{|l|}{ Weight (Kg) } \\
\hline $\begin{array}{r}\text { Mean (SD) } \\
\text { Range }\end{array}$ & $\begin{array}{c}62.0(16.9) \\
9-88\end{array}$ \\
\hline \multicolumn{2}{|l|}{ Height $(\mathrm{Cm})$} \\
\hline $\begin{array}{r}\text { Mean (SD) } \\
\text { Range }\end{array}$ & $\begin{array}{c}162.0(8.3) \\
96-185\end{array}$ \\
\hline \multicolumn{2}{|l|}{ Ideal body weight (Kg) } \\
\hline Mean (SD) & $47.1(3.4)$ \\
\hline $\begin{array}{ll}\text { Range } \\
\end{array}$ & $40-51.8$ \\
\hline \multicolumn{2}{|l|}{$\begin{array}{l}\text { Creatinine clearance using MDRD equation } \\
(\mathrm{mL} / \mathrm{min} / 1,73)\end{array}$} \\
\hline$>50 \mathrm{~mL} / \mathrm{min} / 1.73$ & $74(78 \%)$ \\
\hline $10-50 \mathrm{~mL} / \mathrm{min} / 1.73$ & $17(18 \%)$ \\
\hline$<10 \mathrm{~mL} / \mathrm{min} / 1.73$ & $4(4 \%)$ \\
\hline \multicolumn{2}{|l|}{$\begin{array}{l}\text { Creatinine clearance using MDRD equation } \\
(\mathrm{mL} / \mathrm{min} / 1.73)\end{array}$} \\
\hline$>50 \mathrm{~mL} / \mathrm{min} / 1.73$ & $65(68 \%)$ \\
\hline $10-50 \mathrm{~mL} / \mathrm{min} / 1.73$ & $28(30 \%)$ \\
\hline$<10 \mathrm{~mL} / \mathrm{min} / 1.73$ & $2(2 \%)$ \\
\hline \multicolumn{2}{|l|}{ Type of Aminoglycosides } \\
\hline Gentamicin & $60(63 \%)$ \\
\hline Amikacin & 35 (37\%) \\
\hline \multicolumn{2}{|l|}{ Type of Infection, n (\%) } \\
\hline Ventilator associated pneumonia & $26(27 \%)$ \\
\hline Central nervous system & $23(25 \%)$ \\
\hline Urinary tract infection & $9(10 \%)$ \\
\hline Abdominal infection & $7(8 \%)$ \\
\hline Other sources (Sepsis) & $6(6 \%)$ \\
\hline Abscess & $4(4 \%)$ \\
\hline Pyelonephritis & $4(4 \%)$ \\
\hline Endocarditis & $4(4 \%)$ \\
\hline Cystitis & $4(4 \%)$ \\
\hline Catheter associated infection & $3(3 \%)$ \\
\hline $\begin{array}{l}\text { Peritonitis } \\
\end{array}$ & $3(3 \%)$ \\
\hline Skin infection & $2(2 \%)$ \\
\hline \multicolumn{2}{|l|}{ Source of infection, $n(\%)$} \\
\hline Community acquired & $34(36 \%)$ \\
\hline Hospital acquired & $61(64 \%)$ \\
\hline
\end{tabular}


In $69 \%$ of the patients, AG was initiated empirically and $31 \%$ of the prescriptions were based on microbiological culture data. Among empirical treatment, $17 \%$ of the prescriptions were non-indicated.

Scores of AGs usage at Nemazee hospital was calculated as 5.9 out of 11 , which meant that in only $54 \%$ of the cases AGs prescription was based on the aforementioned guideline.

Only for $31 \%$ of the patients, microbial culture was collected before treatment, and microbial culture was collected 48-72 hours after initiating therapy with AG for $47 \%$ of them.

In $83 \%$ of the cases AGs indication was correct. In $86 \%$ of patients, the first dose of AG was adjusted according to the patient's SCr.

The route of AG administration was appropriate for all patients, but loading doses were not administered for any of the patients. Also, therapeutic drug monitoring was not performed on any of the patients and dosage adjustments were determined as $\mathrm{mg} / \mathrm{kg}$ dosing of the AG. Despite the differences between calculated creatinine clearance in the two formulas (MDRD and Cockcroft and Gault) in the studied population, there was no significant difference in dosage $(p=0.12)$.

The response to treatment was assessed by clearance of cultures. In $19 \%$ of patients, response to treatment was not observed, and only for half of them appropriate action was taken; for $22 \%$, AG dose was increased and for $78 \%$ of them the antibiotic was changed. Fifty-eight percent of the administered doses were appropriate.

In $21 \%$ of patients, nephrotoxicity was developed. In $60 \%$ of the patients who developed nephrotoxicity, no action was taken and in the remaining, the AG administration was discontinued. In $96 \%$ of patients, $\mathrm{SCr}$ was evaluated every day.

On the first day of AG administration, white blood cells count of $48 \%$ of the studied population was more than $10,000 / \mathrm{ml}$. In contrast, only $28 \%$ of the study population had white blood cells counts above $10,000 / \mathrm{ml}$ at the end of treatment.

The treatment period in this study was 6.6 (SD 4) days, ranging between 2 and 18 days. Table 2 has shown the 11 studied indexes of AGs usage in this study.

\section{DISCUSSION}

Present study was conducted to evaluate the pattern of AGs prescription in 4 ICUs of a referral hospital in Shiraz, Iran. The results showed that the overall adherence of AGs usage to guideline is relatively inappropriate in the ICUs of Nemazee hospital. It seems that absence of pharmacists in the health team, lack of physicians' information about the pharmacokinetic of AGs, and inappropriate training and educational programs for physicians and nurses regarding the correct guideline implementation were the main reasons for non-adherence to AG treatment guidelines. In our study, in only $54 \%$ of cases AGs prescription was based on the guideline proposed by the Department of Clinical Pharmacy of Nemazee Hospital. The main errors in the prescription of AGs were lack of administration of loading dose, inappropriate dosing method, lack of re-evaluation in the case of required dose adjustments, and ignorance of the microbiological data.

In a similar study by Namazi et al., conducted in the internal medicine ward of the same hospital, the usage pattern of amikacin, one of the most widely used AGs, was evaluated. Their results showed that the overall adherence of this drug's usage to the guideline was $48 \%$ and inappropriate dosing method, poor patient monitoring, and ignorance of microbiological data were the most common reasons for the none-adherence to standard guidelines. ${ }^{26}$ In this study, serum concentration of Amikacin was assayed using a Cobas Mira AutoAnalyzer, but in our study the serum concentration of AGs was not assessed. In their study, they concluded that the desired peak and trough concentrations were obtained in $38 \%$ and $45 \%$ of the patients. Therapeutic drug monitoring (TDM) of AGs has become a standard practice in many clinical settings, but no serum level was measured for the patients in our hospital and the AGs dose was determined merely according to the patients' clinical status and some other data, such as the patient's GFR calculated by MDRD or G-C formula, as well as his weight. It was shown that AGs TDM can minimize toxicities, maximize efficacy, and improve health outcomes. $^{27}$

In our study, AGs dosage was calculated according to the conventional method for all patients, and the prescribed dose was correct in $58 \%$ of the patients. Also, conventional method was used to calculate amikacin dosage in Namazi et al. study, but the prescribed dose was correct only in $25 \%$ of the patients. Using pharmacokinetic dosing can reduce the risk of adverse effects, such as nephrotoxicity and also allows administration of a significantly greater cumulative dose. $^{28}$

\begin{tabular}{|l|c|}
\hline \multicolumn{1}{|c|}{ Table 2. Eleven indexes of aminoglycosides use in the study population ( $\mathrm{n}=95)$} & Appropriately Performed \\
\hline & $0 \%$ \\
\hline Loading dose & $100 \%$ \\
\hline Route of administration & $0 \%$ \\
\hline Dose & $58 \%$ \\
\hline Indication & $83 \%$ \\
\hline Considering the patient's renal function before prescribing & $86 \%$ \\
\hline Monitoring serum creatinine level periodically during the treatment & $96 \%$ \\
\hline Evaluation of the microbiological culture before prescription & $31 \%$ \\
\hline Evaluation of microbiological culture 48-72 hours after administration & $47 \%$ \\
\hline Reducing the dose or discontinuation in patients who developed AGs nephrotoxicity & $40 \%$ \\
\hline Increase the dose or change the type of antibiotic if there is no appropriate response & $47 \%$ \\
\hline
\end{tabular}


Also, in two other studies conducted in ICUs of Nemazee hospital, it was reported that the rate of Vancomycin and Colistin use, as two commonly used antibiotics in critically ill patients, were in accordance with standard guidelines in $47.3 \%$ and $67.3 \%$ of the cases, respectively. ${ }^{11,29}$

Adherence to AG guideline was also evaluated in a survey conducted in French healthcare facilities. They observed that in more than one third of patients, AG indications were inappropriate and the primary indication use was concordant with the guidelines in $65.2 \%$ of the cases. Unlike our study, AG serum concentration was measured in this survey and the results showed that only, in $62.9 \%$ of the cases, the AG dose was based on the recommended range. ${ }^{30}$ In another Australian study, appropriateness of the initial dose of gentamicin dose was evaluated based on the local guidelines. Based on the existing hospital guidelines, their study result showed that $66 \%$ of the patients did not receive appropriate gentamicin initial dosage. ${ }^{31}$ An adequate loading dose of AGs is critical to achieve rapid therapeutic drug levels. It was suggested that critically ill patients require larger doses of AGs loading dose due to considerable variation in their pharmacokinetic parameters and also the higher resistance of microorganisms in comparison with none-complicated infections. ${ }^{32}$ Pleural effusion, ascites, mediastinitis and hypoalbuminaemia are associated with the expansion of extracellular space, which result in a lower than desirable Cmax with the usual loading dose. Thus, it is suggested to increase the loading dose in this specific population in order to improve the probability of attaining the target Cmax. ${ }^{33}$ However, administration of the AGs loading dose was one of the issues that was apparently ignored by the clinicians in our hospital. One possible reason for this issue was lack of physicians' information regarding the pharmacokinetic of AGs. It seems that the clinicians were not aware of the necessity of administering loading doses to attain adequate drug concentrations immediately after the antibiotic administration.

In our study only in $31 \%$ of patients, AG administration was based on microbiological laboratory evidence while $69 \%$ of the cases received AGs empirically. It is worth mentioning that short term adjunctive treatment of sepsis with AGs in critically ill patients is suggested in some guidelines. The reasons for this suggestion are the ability of AGs to reduce the risk of inappropriate empirical therapy, sterilize the bloodstream faster and act synergistically with b-lactam antibiotics. ${ }^{19,34}$ However, this approach has never been evaluated in randomized studies. ${ }^{35}$

Currently, many hospitals have developed and implemented local guidelines for the use of antimicrobial agents in the empirical treatment of infections. ${ }^{36}$ A study conducted in the United States showed that limiting the use of antibiotics was considered in $56 \%$ of the newly published guidelines, and also in $81 \%$ of medical teaching centers. ${ }^{37}$ Marquet et al. evaluated the inappropriateness of antibiotic therapy in critically ill patients with bloodstream infections in a systematic review and metaanalysis of 37 articles. The rate of inappropriate empiric antibiotic prescription varied between $14 \%$ and $79 \%$. Approximately half of the studies reported the incidence of $50 \%$ or more. The mortality rates were significantly higher in patients receiving inappropriate antibiotics in studies with outcome parameter 28-day and 60-day mortality. Also, inappropriate antibiotic prescription increased the 30 day and in-hospital mortality rates. ${ }^{38}$

In our study, the results showed that $17 \%$ of $A G$ prescriptions were inappropriate. To some extent, it is similar to the Thuong et al. study stating that the selection of primary antibiotics was appropriate for only $73 \%$ of patients. ${ }^{36}$ Unspecified diagnosis, lack of documentation as to why antibiotics were prescribed for patient, inappropriate microbiological sample collection before prescribing and lack of reviewing the available results, might be the reasons of inappropriate prescription or its continuation.

In this study source of infection in $64 \%$ of the cases was reported to be hospital acquired. In another study on patients who were admitted to the ICU after surgery in France, the origin of infection in 55\% of the cases was community and in $45 \%$ of the cases was hospital. ${ }^{39}$

Obtaining a second microbiological culture 48 to 72 hours after the starting AGs is recommended in order to evaluate patient response to treatment. In our study, only $47 \%$ of patients' cultures were collected from the site of infection at 48-72 hours after AG administration and the decisions for drug prescription were made based on that. One of the reasons for this issue is that the physicians in our center did not believe in the accuracy and precision of microbiological tests and they preferred to prescribe antibiotics according to their own experience and clinical judgement. During the study, nephrotoxicity was developed in $21 \%$ of the patients. The reported incidence of nephrotoxicity varies widely due to variations in study design, toxicity definitions, study population, and concomitant risk factors. A reasonable estimate (depending on definition) might be 10 to 20 percent, even when careful patient selection and close monitoring is performed. ${ }^{40}$

It is recommended that $\mathrm{SCr}$ should be evaluated regularly during AGs treatment. This study was conducted in the ICU and in the ICUs of our hospital the $\mathrm{SCr}$ is measured on a daily basis. Hence, in $96 \%$ of patients, $\mathrm{SCr}$ was measured every day. It seems that daily measurement of $\mathrm{SCr}$ level in hospitalized patients was considered as a routine practice for all patients without considering its changing trend.

In this study, creatinine clearance was calculated with both Cockcroft and Gault (C-G) and MDRD methods. Correct administration of the AGs dosage regimen according to correct dosing method using the two Cockcroft and Gault and MDRD equations was $58 \%$ and $57 \%$, respectively. In one study conducted amongst Iranian adults, the authors concluded that MDRD and C-G formulas are accurate in Iranian adults, but it needs a correction factor. ${ }^{41}$

According to protocols, treatment of AGs-induced nephrotoxicity is basically supportive, including discontinuation of AGs (if possible) and substituting it with another non-nephrotoxic antibiotic. If this is not possible, the dose of AG should be adjusted and the interval between doses should be increased. Use of other nephrotoxic drugs should be avoided. ${ }^{42}$ However, only in $40 \%$ of the cases with nephrotoxicity, administration of AGs was discontinued and no action was taken in the case of 
the remaining patients. In Namazi et al. study, $19 \%$ of the patients developed nephrotoxicity due to amikacin, but only in $50 \%$ of the cases it was discontinued. ${ }^{26}$ Several approaches including prescribing less nephrotoxic AGs when possible, extended interval dosing, therapeutic drug monitoring, using nebulized AGs, morning administration of AGs, daily monitoring of serum creatinine and using novel renal biomarkers such as kidney injury molecule-1 (KIM-1) which identifies AG-induced proximal tubular injury earlier than traditional markers have been suggested to prevent AG-induced nephrotoxicity. ${ }^{43}$

Considering the results of this study, developing a detailed plan is an essential step to improve the AGs usage pattern in the ICUs of our hospital. Clinical pharmacists should be incorporated in this setting to have an active role in prescribing AGs as well as pharmacokinetic consultation in the case of drug prescription for critically ill patients. Also, they can participate in arrangements held periodically for monitoring the utilization of drugs by stating their suggestions. They should be involved in training of medical and other healthcare personnel including physicians and nurses with respect to prescription, usage and administration of AGs. An evidence-based, peer reviewed local guideline should be developed in our ICUs based on the latest guidelines on the use of AGs in treating infections and regularly updated whenever new information becomes available. All the barriers to guideline implementation should be identified, using suitable strategies to overcome these barriers.

\section{Limitations}

Our study was a single center study and the sample size was relatively small. Hence, the results cannot be generalized. The incidence of adverse effects was not studied, since measuring some AGs side effects, such as hearing loss, required special instruments (audiometer) which were not available in these ICUs. Also, the studies that were performed to determine the incidence of adverse drug effects require very large sample sizes. Only the increase in $\mathrm{SCr}$ was evaluated as an indicator of renal toxicity as part of treatment assessment for AGs. On the other hand, the group with nephrotoxicity was at increased risk for toxicity due to other factors and not solely $A G$ exposure.

\section{CONCLUSIONS}

In summary, the results of this study indicated that the overall adherence of AGs usage to guideline is relatively inappropriate in the ICUs of Nemazee hospital. Administration of the AGs loading dose is an issue that has not been taken into account in this center. Additionally, measurement of serum AG concentration was not performed in this hospital. It seems that consumption control and training programs are required to improve the pattern of AG usage in the ICUs of this hospital. Also, the presence of pharmacists in the ICUs of this center and providing pharmacokinetic consultation service can greatly help to increase the adherence of AGs usage to the guidelines.

\section{ACKNOWLEDGMENTS}

The authors wish to thank Mr. H. Argasi at the Research Consultation Center of Shiraz University of Medical Sciences for his invaluable assistance in editing this manuscript.

\section{CONFLICT OF INTEREST}

The authors report no conflicts of interest in this work.

\section{FUNDING}

None.

\section{References}

1. Kollef MH, Sherman G, Ward S, Fraser VJ. Inadequate antimicrobial treatment of infections: a risk factor for hospital mortality among critically ill patients. Chest. 1999;115(2):462-474. https://doi.org/10.1378/chest.115.2.462

2. Ibrahim EH, Sherman G, Ward S, Fraser VJ, Kollef MH. The influence of inadequate antimicrobial treatment of bloodstream infections on patient outcomes in the ICU setting. Chest. 2000;118(1):146-155. https://doi.org/10.1378/chest.118.1.146

3. Shorr AF, Micek ST, Welch EC, Doherty JA, Reichley RM, Kollef MH. Inappropriate antibiotic therapy in Gram-negative sepsis increases hospital length of stay. Crit Care Med. 2011;39(1):46-51. https://doi.org/10.1097/CCM.0b013e3181fa41a7

4. Pillans P, ledema J, Donovan P, Newbery R, Whitehead V, Halliday C, Sheehy R, Springford A, Patterson T. Outcomes in patients with gram-negative sepsis treated with gentamicin. Ther Adv Drug Saf. 2012 Jun;3(3):109-113. https://doi.org/10.1177/2042098612439495

5. Hanberger H, Edlund C, Furebring M, C GG, Melhus A, Nilsson LE, Petersson J, Sjolin J, Ternhag A, Werner M, Eliasson E. Rational use of aminoglycosides--review and recommendations by the Swedish Reference Group for Antibiotics (SRGA). Scand J Infect Dis. 2013;45(3):161-175. https://doi.org/10.3109/00365548.2012.747694

6. Kelly KN, Monson JRT. Hospital-acquired infections. Surgery - Oxford International Edition. 2012;30(12):640-4.

7. Garau J. Impact of antibiotic restrictions: the ethical perspective. Clin Microbiol Infect. 2006;12(Suppl 5):16-24. https://doi.org/10.1111/j.1469-0691.2006.01527.x

8. Arda B, Sipahi OR, Yamazhan T, Tasbakan M, Pullukcu H, Tunger A, Buke C, Ulusoy S. Short-term effect of antibiotic control policy on the usage patterns and cost of antimicrobials, mortality, nosocomial infection rates and antibacterial resistance. J Infect. 2007;55(1):41-48. https://doi.org/10.1016/j.jinf.2007.02.014

9. Fraser A, Paul M, Almanasreh N, Tacconelli E, Frank U, Cauda R, Borok S, Cohen M, Andreassen S, Nielsen AD. Benefit of appropriate empirical antibiotic treatment: thirty-day mortality and duration of hospital stay. Am J Med. 2006;119(11):970-976. https://doi.org/10.1016/..amimed.2006.03.034 
10. Leibovici L, Shraga I, Drucker M, Konigsberger H, Samra Z, Pitlik S. The benefit of appropriate empirical antibiotic treatment in patients with bloodstream infection. J Intern Med. 1998;244(5):379-386. https://doi.org/10.1046//.13652796.1998.00379.x

11. Vazin A, Karimzadeh I, Zand A, Hatami-Mazinani N, Firouzabadi D. Evaluating Adherence of Health-Care Team to Standard Guideline of Colistin Use at Intensive Care Units of a Referral Hospital in Shiraz, Southwest of Iran. Adv Pharm Bull. 2017;7(3):391-397. https://doi.org/10.15171/apb.2017.047

12. Organization WH. Introduction to drug utilization research. Geneve: WHO; 2003.

13. Bae SC. Drug utilization review: economical aspect. J Korean Med Assoc. 2004;47(2):156-60

14. Vazin A, Japoni A, Shahbazi S, Davarpanah MA. Vancomycin utilization evaluation at hematology-oncology ward of a teaching hospital in Iran. Iran J Pharm Res. 2012;11(1):163-170.

15. Seng JJB, Yong MHA, Peh ZX, Soong JL, Tan MH. Appropriateness of vancomycin therapeutic drug monitoring and its outcomes among non-dialysis patients in a tertiary hospital in Singapore. Int J Clin Pharm. 2018;40(5):977-981. https://doi.org/10.1007/s11096-018-0670-4

16. Leone M, Bourgoin A, Cambon S, Dubuc M, Albanèse J, Martin C. Empirical antimicrobial therapy of septic shock patients: adequacy and impact on the outcome. Crit Care Med. 2003;31(2):462-467. https://doi.org/10.1097/01.CCM.0000050298.59549.4A

17. Kumar A, Roberts D, Wood KE, Light B, Parrillo JE, Sharma S, Suppes R, Feinstein D, Zanotti S, Taiberg L. Duration of hypotension before initiation of effective antimicrobial therapy is the critical determinant of survival in human septic shock. Crit Care Med. 2006;34(6):1589-1596. https://doi.org/10.1097/01.CCM.0000217961.75225.E9

18. Paul M, Lador A, Grozinsky-Glasberg S, Leibovici L. Beta lactam antibiotic monotherapy versus beta lactam-aminoglycoside antibiotic combination therapy for sepsis. Cochrane Database Syst Rev. 2014;(1):CD003344. https://doi.org/10.1002/14651858.CD003344.pub3

19. Rhodes A, Evans LE, Alhazzani W, Levy MM, Antonelli M, Ferrer R, et al. Surviving sepsis campaign: international guidelines for management of sepsis and septic shock: 2016. Intensive Care Med. 2017;43(3):304-377. https://doi.org/10.1007/s00134-017-4683-6

20. Falagas ME, Kopterides P. Old antibiotics for infections in critically ill patients. Curr Opin Crit Care. 2007;13(5):592-597. https://doi.org/10.1097/MCC.0b013e32827851d7

21. Taccone FS, Laterre P-F, Spapen H, Dugernier T, Delattre I, Layeux B, De Backer D, Wittebole X, Wallemacq P, Vincent $\mathrm{JL}$. Revisiting the loading dose of amikacin for patients with severe sepsis and septic shock. Crit Care. 2010;14(2):R53. https://doi.org/10.1186/cc8945

22. de Montmollin E, Bouadma L, Gault N, Mourvillier B, Mariotte E, Chemam S, Massias L, Papy E, Tubach F, Wolff M. Predictors of insufficient amikacin peak concentration in critically ill patients receiving a $25 \mathrm{mg} / \mathrm{kg}$ total body weight regimen. Intensive Care Med. 2014;40(7):998-1005. https://doi.org/10.1007/s00134-014-3276-x

23. Gálvez R, Luengo C, Cornejo R, Kosche J, Romero C, Tobar E, Illanes V, Llanos O, Castro J. Higher than recommended amikacin loading doses achieve pharmacokinetic targets without associated toxicity. Int J Antimicrob Agents. 2011;38(2):146-151. https://doi.org/10.1016/.j.jantimicag.2011.03.022

24. Agence française de sécurité sanitaire des produits de santé. Update on good use of injectable aminoglycosides, gentamycin, tobramycin, netilmycin, amikacin. Pharmacological properties, indications, dosage, and mode of administration, treatment monitoring. Med Mal Infect. 2012;42(7):301-308. https://doi.org/10.1016/i.medmal.2011.07.007

25. Khwaja A. KDIGO clinical practice guidelines for acute kidney injury. Nephron Clin Pract. 2012;120(4):c179-c184. https://doi.org/10.1159/000339789

26. Namazi S, Sagheb MM, Hashempour MM. Usage pattern and serum level measurement of amikacin in the internal medicine ward of the largest referral hospital in the south of Iran: a pharmacoepidemiological study. Iran J Med Sci. 2016;41(3):191-199.

27. Roberts JA, Norris R, Paterson DL, Martin JH. Therapeutic drug monitoring of antimicrobials. Br J Clin Pharmacol. 2012;73(1):27-36. https://doi.org/10.1111/j.1365-2125.2011.04080.x

28. Bartal C, Danon A, Schlaeffer F, Reisenberg K, Alkan M, Smoliakov R, Sidi A, Almog Y. Pharmacokinetic dosing of aminoglycosides: a controlled trial. Am J Med. 2003;114(3):194-198. https://doi.org/10.1016/s0002-9343(02)01476-6

29. Vazin A, Birjand MM, Darake M. Evaluation of vancomycin therapy in the adult ICUs of a teaching hospital in southern Iran. Drug Healthc Patient Saf. 2018;10:21-26. https://doi.org/10.2147/DHPS.S149451

30. Robert J, Pean Y, Alfandari S, Bru JP, Bedos JP, Rabaud C, Gauzit R. Application of guidelines for aminoglycosides use in French hospitals in 2013-2014. Eur J Clin Microbiol Infect Dis. 2017;36(7):1083-1090. https://doi.org/10.1007/s10096016-2892-5

31. Leong CL, Buising K, Richards M, Robertson M, Street A. Providing guidelines and education is not enough: an audit of gentamicin use at The Royal Melbourne Hospital. Intern Med J. 2006;36(1):37-42. https://doi.org/10.1111/j.14455994.2006.01002.x

32. Mahmoudi L, Niknam R, Mousavi S, Ahmadi A, Honarmand H, Ziaie S, Mojtahedzadeh M. Optimal aminoglycoside therapy following the sepsis: how much is too much? Iran J Pharm Res. 2013;12(2):261-269.

33. Pagkalis S, Mantadakis E, Mavros MN, Ammari C, Falagas ME. Pharmacological considerations for the proper clinical use of aminoglycosides. Drugs. 2011;71(17):2277-2294. https://doi.org/10.2165/11597020-000000000-00000

34. Hanberger H, Edlund C, Furebring M, G. Giske C, Melhus Å, Nilsson LE, Petersson J, Sjölin J, Ternhag A, Werner M. Rational use of aminoglycosides - review and recommendations by the Swedish Reference Group for Antibiotics (SRGA). Scand J Infect Dis. 2013;45(3):161-175. https://doi.org/10.3109/00365548.2012.747694

35. Ong DS, van Werkhoven $\mathrm{CH}$, Cremer OL, Thwaites GE, Bonten MJ. Is a randomized trial of a short course of aminoglycoside added to $\beta$-lactam antibiotics for empirical treatment in critically ill patients with sepsis justified? Clin Microbiol Infect. 2018;24(2):95-96. https://doi.org/10.1016/i.cmi.2017.09.020 
36. Thuong M, Shortgen F, Zazempa V, Girou E, Soussy CJ, Brun-Buisson C. Appropriate use of restricted antimicrobial agents in hospitals: the importance of empirical therapy and assisted re-evaluation. J Antimicrob Chemother. 2000;46(3):501-508. https://doi.org/10.1093/jac/46.3.501

37. Shahbazi F, Dashti-Khavidaki S. Colistin: efficacy and safety in different populations. Expert Rev Clin Pharmacol. 2015;8(4):423-448. https://doi.org/10.1586/17512433.2015.1053390

38. Marquet K, Liesenborgs A, Bergs J, Vleugels A, Claes N. Incidence and outcome of inappropriate in-hospital empiric antibiotics for severe infection: a systematic review and meta-analysis. Crit Care. 2015;19:63. https://doi.org/10.1186/s13054-015-0795-y

39. Rangel-Frausto MS, Wiblin T, Blumberg HM, Saiman L, Patterson J, Rinaldi M, Pfaller M, Edwards Jr JE, Jarvis W, Dawson J. National epidemiology of mycoses survey (NEMIS): variations in rates of bloodstream infections due to Candida species in seven surgical intensive care units and six neonatal intensive care units. Clin Infect Dis. 1999;29(2):253-258. https://doi.org/10.1086/520194

40. Wargo KA, Edwards JD. Aminoglycoside-induced nephrotoxicity. J Pharm Pract. 2014;27(6):573-577. https://doi.org/10.1177/0897190014546836

41. Atapour A, Elham K, Shahidi S, Najafabadi MM, Hedayati P. Modification of diet in renal disease and CockraftGaultformula accuracy in glomerular filtration rate estimation in Iranian adults. Adv Biomed Res. 2013;2:32. https://doi.org/10.4103/2277-9175.108771

42. Oliveira JFP, Cipullo JP, Burdmann EA. Aminoglycoside nephrotoxicity. Antimicrob Agents Chemother. 2009;53(7):28872891. https://doi.org/10.1128/AAC.01430-08

43. McWilliam SJ, Antoine DJ, Smyth RL, Pirmohamed M. Aminoglycoside-induced nephrotoxicity in children. Pediatr Nephrol. 2017;32(11):2015-2025. https://doi.org/10.1007/s00467-016-3533-z 